V. V. Volkov ${ }^{1}$, Sh. A. Kalandarov ${ }^{1,2, *}$

\title{
INTERPRETATION OF THE LOW-ENERGY FISSION PROCESS IN THE FRAMEWORK OF DINUCLEAR SYSTEM CONCEPTION
}

Submitted to «Particles and Nuclei, Letters»

\footnotetext{
${ }^{1}$ Joint Institute for Nuclear Research, Dubna

${ }^{2}$ Institute of Nuclear Physics, Tashkent

*E-mail: shuhrat@jinr.ru
} 
Волков В.В., Каландаров Ш. А.

Интерпретация процесса низкоэнергетического деления тяжелых ядер

в рамках концепции двойной ядерной системы

Обсуждается возможность интерпретации деления тяжелых ядер как процесса формирования, эволюции и распада двойной ядерной системы. Интерпретация основана на данных о ядерных взаимодействиях, полученных в ядернофизических исследованиях с тяжелыми ионами.

Работа выполнена в Лаборатории теоретической физики им. Н. Н. Боголюбова и Лаборатории ядерных реакций им. Г. Н. Флерова ОИЯИ.

Volkov V. V., Kalandarov Sh. A.

E7-2015-91

Interpretation of the Low-Energy Fission Process

in the Framework of Dinuclear System Conception

The possibility of the interpretation of fission of heavy nuclei as the process of formation, evolution, and decay of a dinuclear system is discussed. The interpretation is based on the nuclear interaction data obtained in heavy-ion nuclear physics investigations.

The investigation has been performed at the Bogoliubov Laboratory of Theoretical Physics and the Flerov Laboratory of Nuclear Reactions, JINR. 


\section{INTRODUCTION}

Atomic nuclei are objects of the nuclear microworld, and processes in them are described by quantum mechanics. Quantum mechanics has allowed interpretation and description of numerous phenomena of the microworld, which were impossible within classical mechanics. At the same time, some processes occurring in atomic nuclei are difficult to describe within quantum mechanics. One of such processes is fission of uranium discovered by Hahn and Strassman in 1938. Nuclear fission could not be regarded as a simple quantum transition. It was an evolutionary process in which the initial heavy nucleus passed through many intermediate states ultimately evolving into two lighter fragments.

Just a few months after the discovery of fission, an article was published by N. Bohr and J.A. Wheeler entitled "The Mechanism of Nuclear Fission" [1] in which fission process was described in the framework of the classical liquid-drop model. It should be noted that in the very same article, but in the framework of quantum mechanics, the authors predicted the possibility of spontaneous fission of ${ }^{238} \mathrm{U}$ and estimated the half-life period.

Over a period of nearly thirty years, the fission model proposed by N. Bohr and J.A. Wheeler was a conventional theoretical approach to describing many different aspects of low-energy fission of heavy nuclei. However, this model had one serious drawback: the model predicted symmetric mass distribution of fission products, whereas the experiment clearly indicated its asymmetry.

Following the development of the shell model of nucleus, the shell structure of nuclei was promptly found to generate the asymmetry of mass distribution of fission products. With the efforts of many theoreticians, among whom one should first of all mention V.M. Strutinsky, the macroscopic-microscopic model of fission was developed. The liquid-drop model of nucleus is used to perform calculations on changes in the macroscopic form of nucleus during fission, and shell corrections take account of influence of the shell structure on the potential energy of a fissioning nucleus. Although the combination of the classical and quantum mechanics in this way seems to be artificial, this "physical centaur" was efficiently used in calculations of numerous properties of low-energy fission. In his final report at the international conference devoted to the 50th anniversary of the discovery of fission, J.R. Nix described the macroscopic-microscopic model 
of fission in the following way: "This method synthesizes the best features of two complementary approaches: the smooth trends of the potential energy (with respect to particle number and deformations) are taken from a macroscopic model, and the local fluctuations are taken from a microscopic model" [2].

Despite the success of the macro-microscopic model of fission, we should definitely continue searching for other perceptions of the mechanism of lowenergy fission in the hope to get a better understanding and uncover a more realistic mechanism of this nuclear process.

Nuclear physics investigations with heavy ions revealed two new objects in the nuclear microworld and a novel evolutionary nuclear process. In 1960 Almqvist, Bromley, and Kuehner discovered the nuclear molecule [3] while studying the interaction of two ${ }^{12} \mathrm{C}$ nuclei. In the nuclear molecule, two nuclei come into a very close contact with each other but do not fuse into a compound nucleus, thus, retaining their individuality for a long time. The discovery of deep inelastic transfer reactions (DITRs) [4,5] revealed that, when two nuclei with the energy higher than the Coulomb barrier collide, one observes full dissipation of kinetic energy which turns into inner excitation of collided nuclei and formation of a specific nuclear complex, a dinuclear system (DNS) [4]. In DNS both nuclei interact intensively, exchanging with weakly bound nucleons in outer shells and, at the same time, retain their individuality, which is due to highly bound nucleons in lower shells. In contrast to nuclear molecules, DNS states are not stable. It quickly evolves in time by transferring nucleons from one nucleus to the other. The evolution of DNS is governed by its potential energy, which is a function of its charge and mass asymmetry. If nucleons are transferred from the light nucleus to the heavy one, the evolution of DNS is completed by a compound nucleus being formed. If nucleons are transferred in the opposite direction, a symmetric DNS is formed. For the massive DNS, symmetry proves to be unstable: the DNS nuclei undergo deformation, and the system breaks up into two nuclei fragments, that is, quasi-fission occurs.

DITR allowed us to obtain unique information on how the evolutionary process proceeds in nuclear systems. The point is that both fission and its reverse nuclear process, i.e., nuclear fusion of two nuclei into a compound nucleus, are closed nuclear reactions. Experimenters can only observe the initial states and final products of the process: two fission fragments or the decay products of an excited compound nucleus. Neither the fissioning nucleus nor two colliding nuclei "send out signals" that could give us a realistic picture of these two nuclear processes. The uniqueness of DITRs lays in the fact that they reveal the essence of evolutionary nuclear processes which lead to a deep reconstruction of the fissioning heavy nucleus or two nuclei fusing into a compound nucleus.

Atomic nuclei consist of protons and neutrons. One of the most natural ways of rearrangement of nucleonic structure is ejecting or attaching nucleons. However, nucleus can eject or attach nucleons only when it is in a close contact 
with another nucleus. From the consideration above, we can draw the following fundamental conclusion: the indispensable condition of deep reconstruction of the structure of a nucleus or a complex of two nuclei is the formation of a dinuclear system.

The DNS formation is apparent in complete fusion and quasi-fission reactions, where two nuclei are collided, the kinetic energy of the collision, thus, transforms into the inner excitation of nuclei, whereas nuclei themselves are strongly bound due to the nucleus-nucleus potential. However, in fission process we deal with an excited heavy nucleus. How is DNS formed in this case? The answer is prompted by cluster radioactivity. Analyzing the mechanism of cluster radioactivity, authors of [6] came to the following conclusion: nuclei heavier than ${ }^{208} \mathrm{~Pb}$ are able to spontaneously condense valence nucleons which are above the closed proton and neutron shells of ${ }^{208} \mathrm{~Pb}$, playing the role of a core, to light nuclei-clusters. Condensation of all the valence nucleons into a cluster, which is true for light isotopes of radium and actinides, is accompanied by formation of an asymmetric nuclear molecule. We assume that the capability of heavy nuclei to spontaneously condense valence nucleons to the light nuclei-clusters is inherent to all nuclei heavier than ${ }^{208} \mathrm{~Pb}$. However, for heavier isotopes, not all valence nucleons are condensed into cluster because the process is not energetically favorable. The motion of some of them embraces both nuclei, which gives rise to a covalent bond. The asymmetric nuclear molecule with covalent neutrons essentially constitute a dinuclear system.

\section{MECHANISM OF LOW-ENERGY FISSION OF HEAVY NUCLEI}

In macro-microscopic model, nuclear fission is a process occurring in deformation space. Owning to the excitation energy, a heavy nucleus becomes deformed. With the evolution of deformation, the nucleus passes over the fission barrier. Subsequently, the heavy nucleus decays into two fission fragments, since deformation becomes irreversible. In our interpretation, the fission process evolves in mass and charge asymmetry coordinates of DNS. It consists of three stages. At the first stage, an excited asymmetric DNS is formed as a result of condensation of valence nucleons of the heavy nucleus into a cluster. The second stage involves evolution of DNS by transferring nucleons from heavy to the light nucleus. The DNS evolution ends when its potential energy is at a minimum. In this configuration, one of the DNS nuclei is in the vicinity of the doubly magic nucleus ${ }^{132} \mathrm{Sn}$. The nucleus-nucleus potential in this case is characterized by the potential "pocket" which prevents DNS nuclei from decay. It means that DNS is in a quasi-equilibrium state. It is this property of DNS which determines the statistical character of its decay. The shell structure of nuclei, representing future fragments, and their individual deformations play an important role in the 
formation of the mass, charge, and energy distributions of fission fragments. The statistical decay of DNS from the equilibrium configuration is the third stage of low-energy fission. Our interpretation of the low-energy fission process allows one to understand why the statistical model of fission proposed by Fong [7] is successful in explaining asymmetric mass distributions of fission products of ${ }^{236} \mathrm{U}$ and ${ }^{240} \mathrm{Pu}$. Fong assumed statistical equilibrium at prescission configuration of a fissioning nucleus, which at that time seemed quite unrealistic. Subsequently, an assumption of rather long-lived prescission configuration was made in [8]. It allowed use of the statistical approach to describing the prescission configuration decay into different exit channels of fission. The influence of the shell structure and deformation of fragments on the decay probability was taken into account. As a consequence, the results were in satisfactory agreement with the experimental data on mass, charge, and energy distributions of fission fragments. The interpretation of the prescission configuration of a fissioning nucleus as DNS was for the first time proposed in $[9,10]$.

Certain information on the fission mechanism can be obtained from comparison of fission and fusion processes. These two reactions can be regarded as the direct and reverse processes, proceeding through similar intermediate states. This comparison was made in [11]. The author and his colleagues developed in their works a model of complete fusion of nuclei based on the DNS conception [12]. The practice has proven that this conception presents the most realistic picture of the mechanism of complete fusion of nuclei [13]. In [14], it was shown experimentally that at the last stage of fusion, DNS adopts the form of asymmetric nuclear molecules in which lighter nuclei are ${ }^{16} \mathrm{O},{ }^{15} \mathrm{~N},{ }^{12} \mathrm{C}$, and ${ }^{4} \mathrm{He}$. The system conceivably could also go through these DNS configurations, but in a reverse order, at the first stage of fission of heavy nuclei.

\section{POTENTIAL ENERGY OF DINUCLEAR SYSTEM FORMED IN LOW-ENERGY FISSION PROCESS}

The potential energy of DNS is calculated as follows [15]:

$$
U(R, Z, A)=B_{1}+B_{2}+V\left(R, Z, A, \beta_{1}, \beta_{2}\right)-B_{12},
$$

where $B_{1}$ and $B_{2}$ are the mass excesses of the fragments at their ground states, $Z$ and $A$ are charge and mass number of one of the nuclei of DNS, $\beta_{1}$ and $\beta_{2}$ are their quadrupole deformation parameters which are taken from [16] for even-even nuclei. For the quadrupole deformation parameter of odd nucleus, we choose the maximal value from the deformation parameters of neighboring even-even nuclei. The experimental values of $B_{1}$ and $B_{2}$ are used, if available in [17]. Otherwise, we use the values from [18]. Here, $B_{12}$ is the mass excess 
of $\mathrm{CN}$. The nucleus-nucleus potential

$$
V\left(R, Z, A, \beta_{1}, \beta_{2}\right)=V_{C}\left(R, Z, A, \beta_{1}, \beta_{2}\right)+V_{N}\left(R, Z, A, \beta_{1}, \beta_{2}\right)
$$

in Eq. (1) is the sum of the Coulomb potential $V_{C}$ and the nuclear potential $V_{N}$. For calculation of the Coulomb potential we applied a well-known Wongs formula [19]. For the nuclear part of the nucleus-nucleus potential, we use the double-folding formalism [15]

$$
V_{N}=\int \rho_{1}\left(\mathbf{r}_{1}\right) \rho_{2}\left(\mathbf{R}-\mathbf{r}_{\mathbf{2}}\right) F\left(\mathbf{r}_{1}-\mathbf{r}_{\mathbf{2}}\right) d \mathbf{r}_{1} d \mathbf{r}_{\mathbf{2}}
$$

where $F\left(\mathbf{r}_{\mathbf{1}}-\mathbf{r}_{\mathbf{2}}\right)=C_{0}\left[F_{\mathrm{in}} \frac{\rho_{0}\left(\mathbf{r}_{\mathbf{1}}\right)}{\rho_{00}}+F_{\mathrm{ex}}\left(1-\frac{\rho_{0}\left(\mathbf{r}_{\mathbf{1}}\right)}{\rho_{00}}\right)\right] \delta\left(\mathbf{r}_{\mathbf{1}}-\mathbf{r}_{\mathbf{2}}\right)$ is the Skyrme-type density-depending effective nucleon-nucleon interaction, which is known from the theory of finite Fermi systems [20], and $\rho_{0}(\mathbf{r})=\rho_{1}(\mathbf{r})+\rho_{2}(\mathbf{R}-\mathbf{r}), F_{\text {in,ex }}=$ $f_{\text {in,ex }}+f_{\text {in,ex }}^{\prime} \frac{\left(N_{1}-Z_{1}\right)\left(N_{2}-Z_{2}\right)}{\left(N_{1}+Z_{1}\right)\left(N_{2}+Z_{2}\right)}$. Here, $\rho_{1}\left(\mathbf{r}_{1}\right)$ and $\rho_{2}\left(\mathbf{r}_{2}\right)$, and $N_{2}\left(Z_{2}=A_{2}-N_{2}\right)$ are the nucleon densities of the light and heavy nuclei of DNS and neutron (charge) number of the heavy nucleus of DNS, respectively. Our calculations are performed with the following set of parameters: $C_{0}=300 \mathrm{MeV} \cdot \mathrm{fm}^{3}, f_{\text {in }}=0.09$, $f_{\text {ex }}=-2.59, f_{\text {in }}^{\prime}=0.42, f_{\text {ex }}^{\prime}=0.54$, and $\rho_{00}=0.17 \mathrm{fm}^{-3}[20]$.

The densities of the nuclei are taken in the two-parameter symmetrized Woods-Saxon form. Radius parameter $r_{0}$ in nuclear densities is taken as $1.2 \mathrm{fm}$ for all nuclei, except for alpha particle for which we take $1.02 \mathrm{fm}$. Diffuseness parameter is parameterized here as $a_{0}=0.54+(0.011 Z)^{2}(A-2 Z) / A$, to take effectively into account deformation and isospin effects on nuclear densities during fission process.

Due to the sum of the repulsive Coulomb and attractive nuclear forces in Eq. (2), the nucleus-nucleus potential has a "pocket" with a minimum situated for pole-pole orientation at the touching distance between the nuclei $R=R_{m} \approx$ $R_{1}\left(1+\sqrt{5 /(4 \pi)} \beta_{1}\right)+R_{2}\left(1+\sqrt{5 /(4 \pi)} \beta_{2}\right)+0.5 \mathrm{fm}$, where $R_{i}=r_{0} A_{i}^{1 / 3}$ are the radii of interacting nuclei. The DNS is localized at the minimum of this pocket. The position of the Coulomb barrier in $V$ approximately corresponds to $R=R_{b} \approx R_{m}+2 \mathrm{fm}$ in DNS under consideration. Then the depth of the potential pocket is $d_{\text {pot }}=V\left(R_{b}, Z_{1}, A_{1}, \beta_{1}, \beta_{2}\right)-V\left(R_{m}, Z_{1}, A_{1}, \beta_{1}, \beta_{2}\right)$. The depth of the potential pocket depends on the charge asymmetry of DNS. For the asymmetric DNS, the potential pocket is deeper than the one for a more symmetric configuration.

In the case of thermal neutron-induced reaction, excitation energy of $\mathrm{CN}$ is

$$
E_{\mathrm{CN}}^{*}=Q
$$

where $Q=B_{12}-B_{1}-B_{2}$. Excitation energy of DNS can be calculated from $E_{\mathrm{CN}}^{*}$ as follows:

$$
E_{\mathrm{DNS}}^{*}=E_{\mathrm{CN}}^{*}-U(R, Z, A) .
$$




\section{INTERPRETATION OF SOME PROPERTIES OF LOW-ENERGY FISSION}

Let us consider some properties of low-energy fission within the proposed interpretation of this nuclear process. In the interpretation of fission mechanism suggested in this work, some new definitions arise for quantities which usually describe fission process. Let us demonstrate these new features of fission process by way of examples. In Figs. 1-4, we present the potential energy of DNS as a function of its charge asymmetry coordinate for the nuclei ${ }^{233} \mathrm{Th},{ }^{239} \mathrm{U},{ }^{236} \mathrm{U}$, and ${ }^{240} \mathrm{Pu}$, which are formed in thermal neutron capture by the nuclei ${ }^{232} \mathrm{Th},{ }^{238} \mathrm{U}$, ${ }^{235} \mathrm{U}$, and ${ }^{239} \mathrm{Pu}$. In Figs. 1 and 2, one can see that excitation energy of DNS, in the case of ${ }^{233} \mathrm{Th}$ and ${ }^{239} \mathrm{U}$, is not sufficient to overcome the maximum of the potential energy. It means that capture of the thermal neutron followed by fission of these nuclei will not occur. On the contrary, ${ }^{236} \mathrm{U}$ and ${ }^{240} \mathrm{Pu}$ (Figs. 3 and 4) prove to be excited enough for DNS to overcome the maximum of the potential barrier and fall into the "valley" of evolution which leads to the fissioning configuration. Thus, the maximum of the driving potential determines the energy threshold (or fission barrier) of fission process. During nucleon exchange process, we assume that DNS has no time to develop deformations to adjust to the energy minimum. Thus, we took deformations of DNS nuclei in their ground states. However, we believe that deformations will develop at the third stage of the fission process, i.e., during the separation phase, thus, affecting the kinetic energies of future fragments.

In Figs. 5 and 6, we present the nucleus-nucleus potential for DNSs formed from the nuclei ${ }^{236} \mathrm{U}$ and ${ }^{240} \mathrm{Pu}$. These DNSs reached the minimum of potential

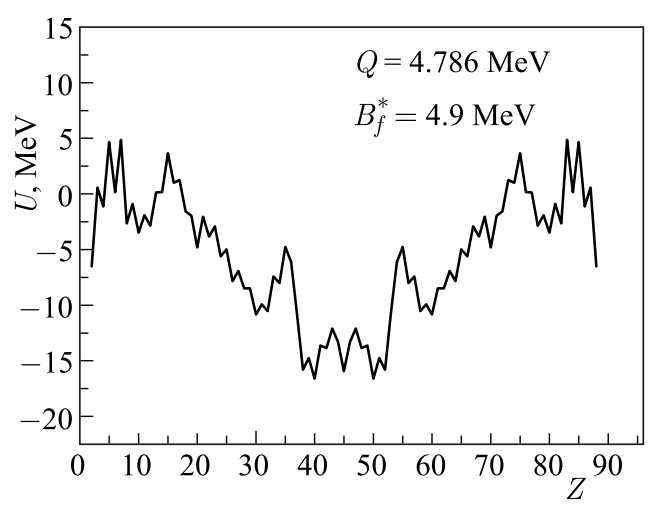

Fig. 1. Potential energy of DNS, formed in $n$ (thermal) $+{ }^{232}$ Th reaction, in dependence on charge asymmetry coordinate of the system. The value of $A$ relates to $Z$ to supply the minimum of $U . Z$ is the charge number of one DNS nuclei, $B_{f}^{*}$ is fission threshold, $Q$ is the $Q$-value of the reaction 


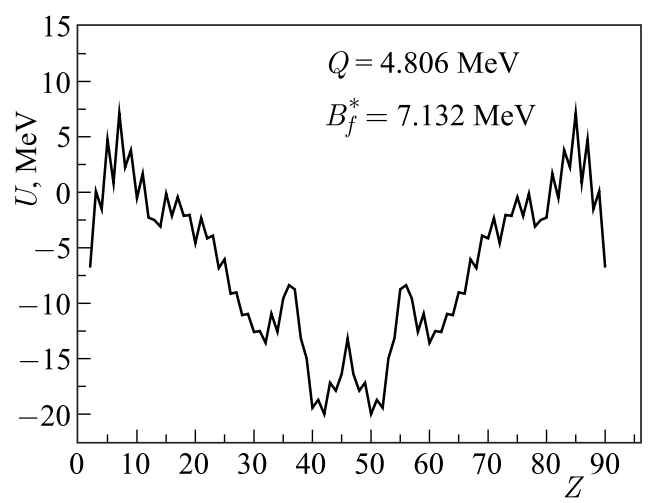

Fig. 2. The same as in Fig. 1, but for $n$ (thermal) $+{ }^{238} \mathrm{U}$ reaction

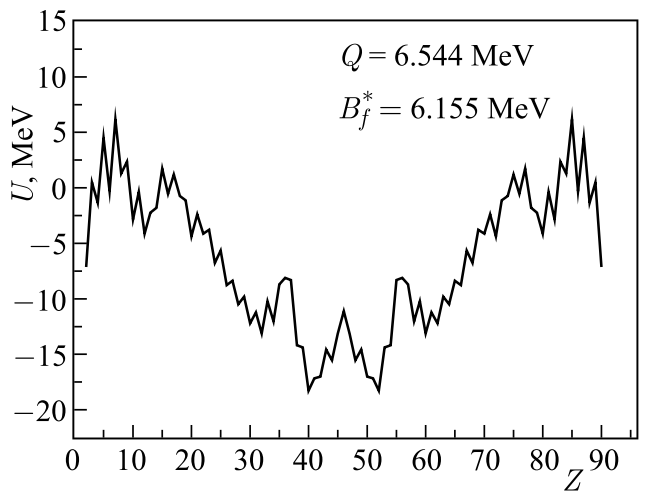

Fig. 3. The same as in Fig. 1, but for $n$ (thermal) $+{ }^{235} \mathrm{U}$ reaction

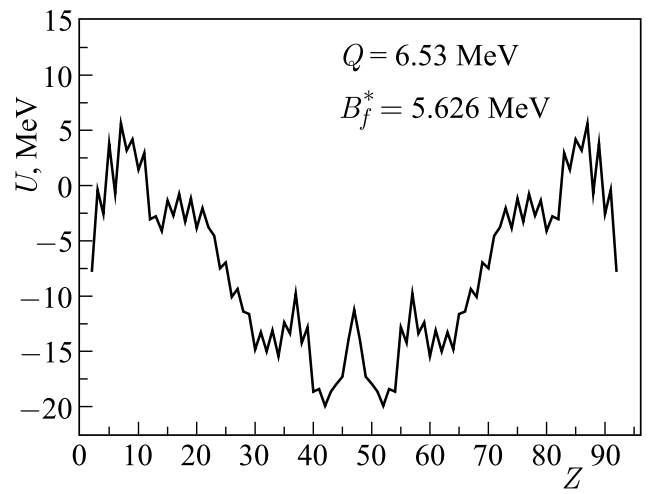

Fig. 4. The same as in Fig. 1, but for $n$ (thermal) $+{ }^{239} \mathrm{Pu}$ reaction 


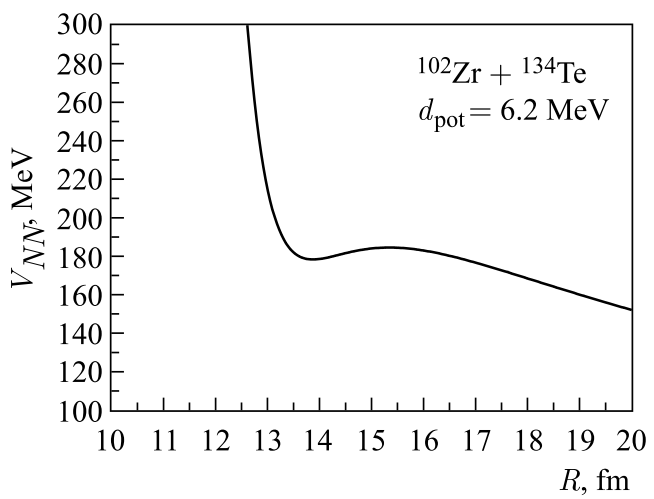

Fig. 5. Nuclear-nuclear potential of DNS in equilibrium configuration for $n$ (thermal) + ${ }^{235} \mathrm{U}$ reaction. $d_{\text {pot }}$ is the depth of potential pocket

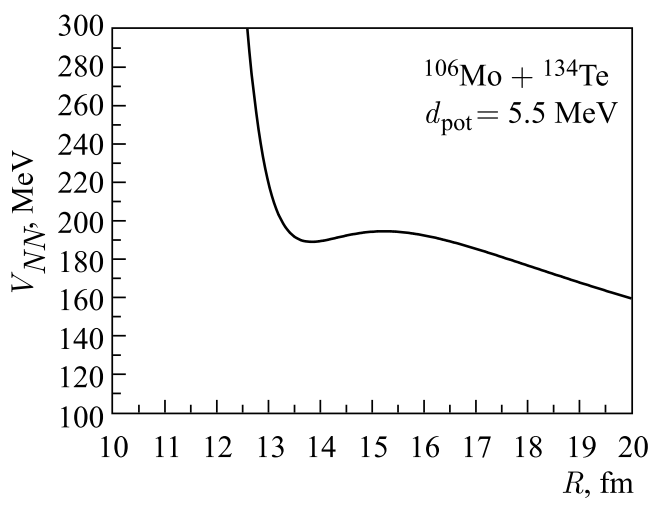

Fig. 6. The same as in Fig. 5, but for $n$ (thermal) $+{ }^{239} \mathrm{Pu}$ reaction

energy. One can see that in both cases nuclear attraction prevails over the Coulomb repulsion, which means that DNS is in a quasi-stationary state and cannot decay instantly. It is the duration of lifetime of the equilibrium DNS in nuclear timescale that makes it possible to reveal the statistical behavior during its decay. This is also supported by successful description by Andreev et al. of the mass, charge, and energy distributions of low-energy fission fragments with the improved statistical scission-point model [21,22]. Quasi-stationary equilibrium of the prescission configuration of DNS is also confirmed by the recent discovery of the third source of fission neutrons [23], which are believed to be related to the prescission configuration of the fissioning nucleus.

In Fig. 7, we show the excitation energy of DNS and the depth of the potential "pocket", varying with the charge asymmetry of the system formed in 


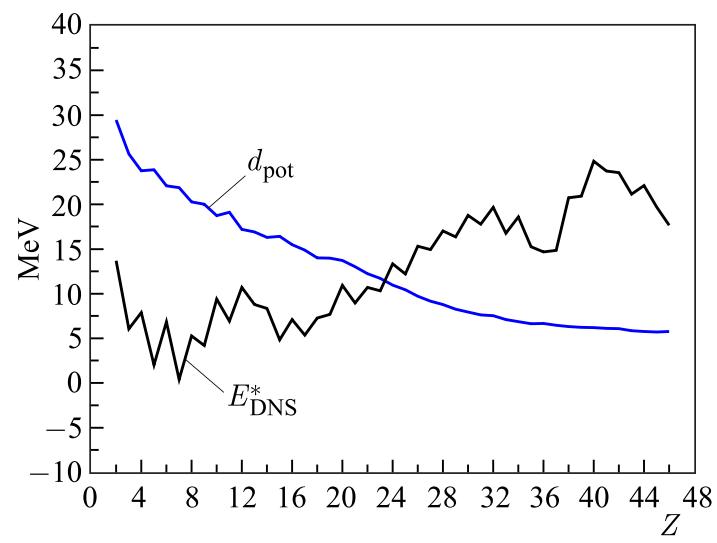

Fig. 7. Excitation energy and $d_{\text {pot }}$ of DNS configurations formed in $n$ (thermal) $+{ }^{235} \mathrm{U}$ reaction

the $n$ (thermal) $+{ }^{235} \mathrm{U}$ reaction. One can see that the excitation energy of DNS exceeds the depth of the potential "pocket" when the system reaches the charge asymmetry $Z \approx 24$. It means that the system can in principle partially decay before reaching the equilibrium configuration, and the dynamics of motion in charge/mass asymmetry coordinates can affect the mass and charge distributions of fission fragments. On the other hand, it does not seem possible to concentrate all the energy in only one collective degree of freedom which leads to the decay of the system. Therefore, this question will be the subject of our future investigations.

\section{CONCLUSIONS}

The interpretation of low-energy fission of heavy nuclei within the dinuclear system (DNS) conception is suggested. The interpretation is based on the information on the properties of nuclei and nuclear processes, which is obtained in nuclear physics investigations with accelerated heavy ions.

The process of low-energy fission comprises three stages. At the first stage, an excited asymmetric DNS is formed as a result of condensation of valence nucleons of the heavy nucleus (nucleons located above the closed proton and neutron shells of ${ }^{208} \mathrm{~Pb}$ ) into a light nucleus-cluster. The second stage involves evolution of DNS by transferring nucleons from the heavy to the light nucleus. The DNS evolution ends when its potential energy is at a minimum. In this configuration, one of the DNS nuclei is in the vicinity of the doubly magic nucleus ${ }^{132} \mathrm{Sn}$. In equilibrium configuration, nuclear attraction prevails over the Coulomb repulsion, and the excited DNS is in a quasi-stationary state. 
The statistical decay of DNS from the equilibrium configuration is the third stage of low-energy fission. The decay of DNS is induced by deformation of DNS nuclei due to the Coulomb forces. The probability of the DNS decay is determined by statistical laws, where in calculation of decay barriers the shell structure of DNS nuclei and their deformations play an important role.

In our interpretation, fission threshold has a new definition. Fission thresholds for ${ }^{233} \mathrm{Th},{ }^{239} \mathrm{U},{ }^{236} \mathrm{U}$, and ${ }^{240} \mathrm{Pu}$ derived from our model describe well the fissionability of these nuclei by thermal neutrons.

Moreover, we give a possible explanation of why statistical models are efficient in describing the mass, charge, and energy distributions of fission fragments observed in experiments.

Acknowledgements. This work was partially supported by RFBR.

\section{REFERENCES}

1. Bohr N., Wheeler J. A. The Mechanism of Nuclear Fission // Phys. Rev. 1939. V. 56. P. 426.

2. Nix J. R. Summary: Our 50-Year Odyssey with Fission // Nucl. Phys. A. 1989. V. 502. P. 609c.

3. Almqvist E., Bromley D. A., Kuehner J. A. Resonances in ${ }^{12} \mathrm{C}$ on Carbon Reactions // Phys. Rev. Lett. 1960. V.4. P.515.

4. Volkov V. V. Deep Inelastic Transfer Reactions - the New Type of Reactions between Complex Nuclei // Phys. Rep. 1978. V.44. P. 93.

5. Schröder W. V., Huizenga J. R. Damped Nuclear Reactions // Treatise on Heavy-Ion Science / Ed.: D. A. Bromley. New York; London, 1984. V. 2. P. 115.

6. Volkov V. V., Cherepanov E. A. Formation of Nuclear Molecules in Cluster Radioactivity // Part. Nucl., Lett. 2013. V. 10, No.3(180). P. 347.

7. Fong P. Statistical Theory of Nuclear Fission. New York: Gordon and Breach, 1969.

8. Wilkins B. D., Steinberg E. P., Chasman R. R. Scission-Point Model of Nuclear Fission Based on Deformed-Shell Effects // Phys. Rev. C. 1976. V. 14. P. 1832.

9. Hulet E. K. et al. Spontaneous Fission Properties of ${ }^{258} \mathrm{Fm},{ }^{259} \mathrm{Md},{ }^{260} \mathrm{Md},{ }^{258} \mathrm{No}$, and ${ }^{260}[104]$ : Bimodal Fission // Phys. Rev. 1989. V. 40. P. 770; Bimodal Symmetric Fission Observed in the Heaviest Elements // Phys. Rev. Lett. 1986. V. 56. P. 313.

10. Andreev A. V. et al. Possible Explanation of Fine Structures in Mass-Energy Distribution of Fission Fragments // Eur. Phys. J. A. 2004. V.22. P. 51.

11. Volkov V. V. Role of Dinuclear System in Complete Fusion Reactions and in Fission of Excited Compound Nuclei // Proc. of the Intern. School-Seminar on Heavy Ion Physics. Dubna, 1989.

12. Antonenko N. V. et al. Competition between Complete Fusion and Quasi-Fission in Reactions between Massive Nuclei. The Fusion Barrier // Phys. Lett. B. 1993. V. 319. P. 425; Compound Nucleus Formation in Reactions between Massive Nuclei: Fusion Barrier // Phys. Rev. C. 1995. V.51. P. 2635. 
13. Volkov V. V. Process of Complete Fusion of Nuclei // Phys. Part. Nucl. 2004. V. 35. P. 425.

14. Volkov V. V. Centrifugal Fragmentation of a Dinuclear System in the Process of Its Evolution to the Compound Nucleus // Phys. Atom. Nucl. 2007. V.70. P. 2046.

15. Adamian G. G. et al. Potential Energy of Dinuclear System // Int. J. Mod. Phys. E. 1996. V.5. P. 191.

16. Raman S., Nestor C.W., Tikkanen P. Transition Probability from the Ground to the First Excited $2^{+}$State of Even-Even Nuclides // At. Data Nucl. Data Tables. 2001. V.78. P. 1.

17. Audi G., Wapstra A.M., Thibault C. The Ame2003 Atomic Mass Evaluation: (II). Tables, Graphs and References // Nucl. Phys. A. 2003. V.729. P. 337.

18. Möller P. et al. Nuclear Ground-State Masses and Deformations // At. Data Nucl. Data Tables. 1995. V.59. P. 185.

19. Wong C. Y. Interaction Barrier in Charged-Particle Nuclear Reactions // Phys. Rev. Lett. 1973. V.31. P. 12.

20. Migdal A. B. Theory of Finite Fermi System and Properties of Atomic Nuclei. Moscow: Nauka, 1983.

21. Andreev A. V. et al. Bimodality and Charge Splitting in Fission of Actinides // Eur. Phys. J. A. 2005. V.26. P. 327.

22. Andreev A. V., Adamian G. G., Antonenko N. V. Mass Distributions for Induced Fission of Different Hg Isotopes // Phys. Rev. C. 2012. V. 86. P. 044315.

23. Svirin M.I. Abstract for a Doctorate Degree in Physics and Mathematics. Dubna, 2014.

Received on October 20, 2015. 


\section{Редактор Е. И. Крупко}

Подписано в печать 16.11.2015.

Формат $60 \times 90 / 16$. Бумага офсетная. Печать офсетная.

Усл. печ. л. 0,9. Уч.-изд. л. 1,2. Тираж 240 экз. Заказ № 58674.

Издательский отдел Объединенного института ядерных исследований 141980 , г. Дубна, Московская обл., ул. Жолио-Кюри, 6.

E-mail: publish@jinr.ru www.jinr.ru/publish/ 